\title{
Time-efficient and contrast-free magnetic resonance imaging approach to the diagnosis of deep vein thrombosis on black-blood gradient-echo sequence: a pilot study
}

\author{
Huan Mao ${ }^{1 \#}$, Xiuhong Guan ${ }^{1 \#}$, Kewen Peng ${ }^{2}$, Yanjun Cai ${ }^{1}$, Jing Yang ${ }^{1}$, Xueping He ${ }^{3}$, Hanwei Chen ${ }^{3}$, \\ Xiaoyong Zhang ${ }^{4}$, Xiaoming $\mathrm{Bi}^{5}$, Xin $\mathrm{Liu}^{6}$, Debiao $\mathrm{Li}^{7}$, Zhaoyang Fan ${ }^{7}$, Zhixian Deng ${ }^{1}$, Guoxi Xie ${ }^{1}$ \\ ${ }^{1}$ Department of Biomedical Engineering, The Sixth Affiliated Hospital, School of Basic Sciences, Guangzhou Medical University, Guangzhou, \\ China; ${ }^{2}$ Department of Radiology, Nanshan People's Hospital, Shenzhen, China; ${ }^{3}$ Department of Radiology, Panyu Central Hospital, Guangzhou, \\ China; ${ }^{4}$ MR Collaborations, Siemens Healthcare Ltd., Shenzhen, China; ${ }^{5}$ MR R\&D, Siemens Healthineers, Los Angeles, CA, USA; ${ }^{6}$ Paul C. \\ Lauterbur Biomedical Imaging Center, Shenzhen Institutes of Advanced Technology, Shenzhen, China; ${ }^{7}$ Biomedical Imaging Research Institute, \\ Cedars Sinai Medical Center, Los Angeles, CA, USA \\ \#These authors contributed equally to this work.
}

Correspondence to: Guoxi Xie, PhD. Department of Biomedical Engineering, The Sixth Affiliated Hospital, Guangzhou Medical University, Xinzao, Panyu District, Guangzhou 511436, China. Email: guoxixie@163.com; Xueping He, MS. Department of Radiology, Panyu Central Hospital, Shiqiao, Panyu District, Guangzhou 511436, China. Email: 978164300@qq.com.

Background: Black-blood thrombus imaging (BTI) has shown to be advantageous for the diagnosis of deep vein thrombosis (DVT). However, previous techniques using fast spin echo have a high specific absorption rate. As DANTE (delay alternating with nutation for tailored excitation) black-blood preparation can suppress blood flows over a broad range of velocities, we hypothesized that a DANTE black-blood preparation combined with a fast low-angle shot (FLASH) gradient-echo readout-DANTE-FLASH could be used to diagnose DVT.

Methods: Eleven healthy volunteers and 30 suspected DVT patients were recruited to undergo DANTEFLASH and magnetic resonance direct thrombus imaging (MRDTI). The suspected DVT patients were also examined by ultrasound (US). For the segment level, a total of 1,066 venous vessel segments were analyzed. Using US and MRDTI as the references, the sensitivity (SE), specificity (SP), positive and negative predictive values (PPV and NPV), and accuracy (ACC) of DANTE-FLASH were calculated. To quantitatively compare image quality between DANTE-FLASH and MRDTI, image signal-to-noise ratio (SNR), apparent contrastto-noise ratio (CNR) between muscle and the venous lumen, and the apparent CNR between the thrombus and venous lumen were measured. Additionally, diagnostic confidence, image quality, and clot burden were also evaluated.

Results: Using the consensus results of US and MRDTI as a standard reference, the diagnostic SE, SP, PPV, NPV, and ACC of DANTE-FLASH for the 2 readers were $97.0 \%$ and $93.2 \%, 99.0 \%$ and $98.2 \%, 93.4 \%$ and $87.9 \%, 99.6 \%$ and $99.0 \%$, and $98.8 \%$ and $97.6 \%$, respectively. According to the image quantitative analysis results, DANTE-FLASH demonstrated higher image SNR and CNR than MRDTI. The image quality and diagnostic confidence scores of DANTE-FLASH were higher than MRDTI (3.66 \pm 0.44 vs. $3.52 \pm 0.52, \mathrm{P}<0.001$, and $3.84 \pm 0.36$ vs. $3.76 \pm 0.41, \mathrm{P}<0.001)$. There was excellent agreement between DANTE-FLASH and MRDTI on clot burden evaluation.

Conclusions: DANTE-FLASH provided better image quality than MRDTI and accurately detected thrombi. It may, therefore, serve as a safe and convenient alternative for the diagnosis of DVT.

Keywords: Deep vein thrombosis (DVT); magnetic resonance imaging; thrombus 
Submitted Sep 10, 2019. Accepted for publication Sep 01, 2020.

doi: 10.21037/qims-19-761

View this article at: http://dx.doi.org/10.21037/qims-19-761

\section{Introduction}

Lower extremity deep vein thrombosis (DVT) has an estimated annual incidence of approximately 1 per 1,000 in the general population, and its incidence increases with age (1). DVT can result in many complications, including pulmonary embolism, post-thrombotic syndrome, and chronic pulmonary embolism associated with pulmonary arterial hypertension (2). The accurate detection of thrombosis is important for DVT treatment decisionmaking and preventing DVT progression (3).

Medical imaging techniques, including ultrasound (US), $\mathrm{X}$-ray, and magnetic resonance (MR) imaging, play essential roles in the diagnosis of DVT (4). They can be used to identify thrombus size and location. US is the currently accepted first-line imaging test for the diagnosis of DVT in the lower extremities (5). Although the US is a noninvasive method that does not involve ionizing radiation or the administration of radiographic contrast agents (6-8), it tends to be susceptible to generating false-negative venous ultrasonography results, particularly in obese patients or patients with markedly edematous limbs (9). Additionally, US probe placement can be difficult in patients with orthopedic casts or those with extensive skin wounds (7).

MR imaging has high resolution in soft tissues, and because it does not use ionizing radiation, it represents an alternative or complementary tool to the US for the diagnosis of DVT (10). Several MR techniques have been developed in the past decades. These include both contrast-enhanced and non-contrast-enhanced MR methods. The former is unsuitable for patients with severe renal insufficiency or pregnancy due to using contrast medium (11). The latter has gained increased attention due to its non-contrast nature. Noncontrast-enhanced MR techniques that can be used for the diagnosis of DVT include MR direct thrombus imaging (MRDTI), blackblood thrombus imaging (BTI), balanced steady-state free precession (bSSFP), and time-of-flight (TOF). The principle underlying using MRDTI to detect thrombosis is from the presence of short-T1 methemoglobin within an acute or subacute thrombus, as it produces a hyperintense signal on the T1-weighted images acquired by a magnetization-prepared gradient echo (GRE) sequence (12). When combined with fat saturation, a hyperintense thrombus can be directly distinguished from the moderateintensity background in T1-weighted images. Unlike MRDTI, the principles underlying using other techniques for detecting thrombosis are based on the finding that a thrombus will present very low intense signals within the hyperintense blood venous lumen (e.g., bSSFP) $(13,14)$, or have intrinsic signal intensity (SI) within the dark venous lumen due to the suppression of venous blood signals (e.g., BTI) (15). Compared to bSSFP and TOF, BTI can be used to visualize the thrombus signals directly. It achieves over $90 \%$ sensitivity (SE), specificity (SP), and accuracy (ACC) in the diagnosis of DVT (15-17). Because BTI is a T1-weighted imaging technique that shares the same mechanism as MRDTI, it may help in thrombus staging (16).

Currently, all BTI techniques are based on threedimensional variable flip angle fast spin echo (3D-vFSE) sequences because of their advantages, including high signal-to-noise ratio (SNR), an intrinsic black-blood effect, and fast data acquisition (15-17). However, 3D-vFSE uses a train of large refocusing flip angles, which results in a high specific absorption rate (SAR). Recently, a time-efficient and contrast-free MRI technique was developed for femoral artery vessel wall imaging in the lower extremities (18). This technique, DANTE-FLASH, uses DANTE (delay alternating with nutation for tailored excitation) for blackblood preparation $(19,20)$ and a low SAR time-efficient fast low-angle shot (FLASH) gradient-echo sequence for data readout. Because DANTE has been demonstrated to be useful for suppressing blood flow over a broad range of velocities above approximately $0.1 \mathrm{~cm} / \mathrm{s}$ (19), we hypothesized that a DANTE preparation would effectively suppress venous blood flow. These findings are much lower than venous blood flow velocity $(9-43 \mathrm{~cm} / \mathrm{s})(21)$, allowing for the low SAR DANTE-FLASH technique to detect thrombi effectively.

Thus, this study was performed to optimize DANTEFLASH for thrombus imaging and investigate its feasibility in the diagnosis of DVT. 


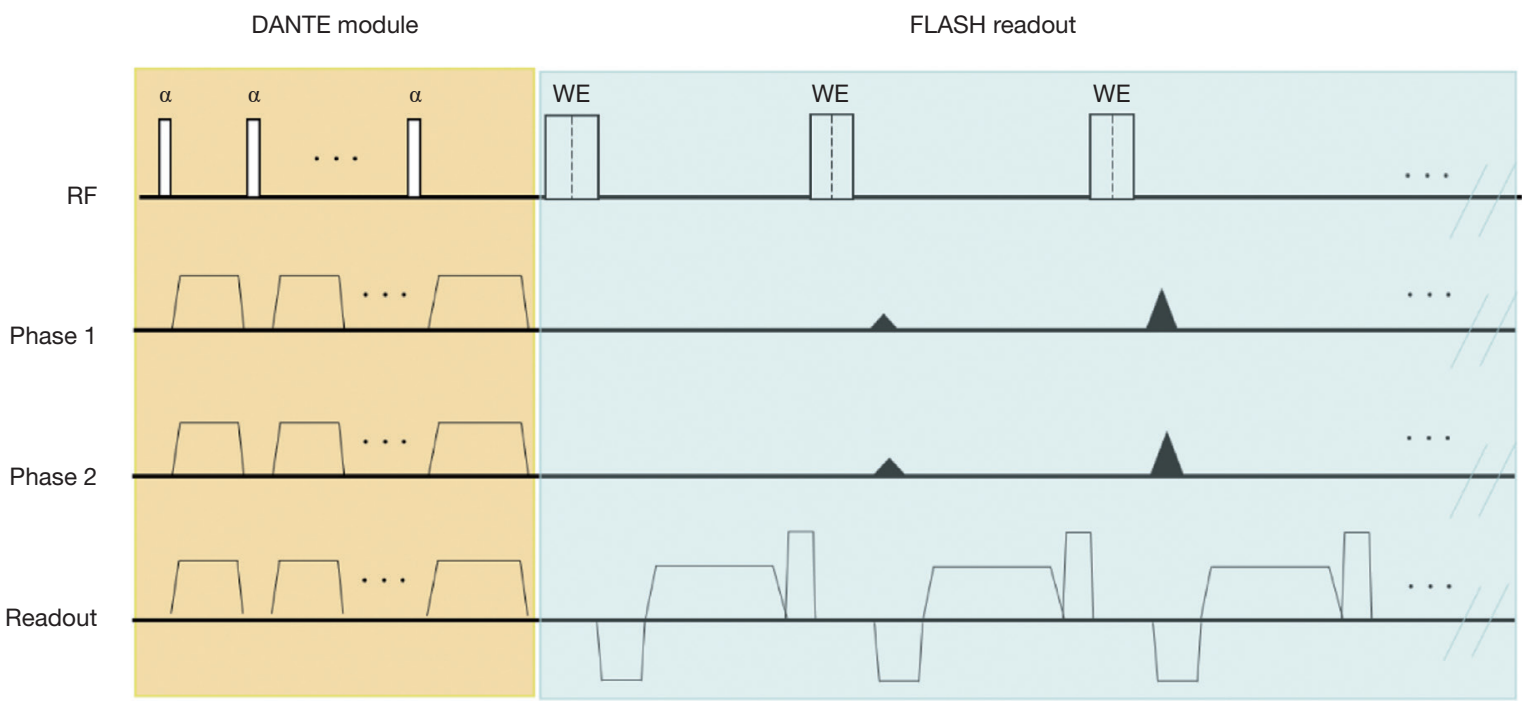

Figure 1 Sequence diagram of DANTE-FLASH for the diagnosis of deep vein thrombosis (DVT). The process consisted of a DANTE module for black-blood preparation, and a low-SAR 3D centrically ordered FLASH readout for data acquisition. Notably, a nonselective water excitation (WE) radiofrequency $(\mathrm{RF})$ pulse was used in the FLASH readout for fat saturation and to shorten echo spacing.

\section{Methods}

\section{Sequence}

The sequence diagram of DANTE-FLASH is shown in Figure 1. It consisted of a DANTE black-blood preparation and a low SAR 3D centric-reordering FLASH readout. Unlike the DANTE-FLASH technique previously used for lower extremity artery vessel wall imaging, two new features were added to this technique for adapting it to thrombus imaging. First, a water excitation (WE) radiofrequency (RF) pulse was used instead of chemical spectral fat saturation (FatSat) in the FLASH readout for fat saturation. This is because fat saturation is more uniform when produced by WE than FatSat (22-24), resulting in better visualization of the image (yellow rectangle regions in Figure 2). Second, a nonselective RF pulse was used instead of a slab selective pulse to achieve shorter echo spacing $(5.4 \mathrm{~ms})$. This shorter echo spacing helps reduce abdominal motion artifacts during imaging of the iliac vessels (red rectangle regions in Figure 2).

\section{Subjects}

The local institutional review board approved this study, and written consent was obtained from all participants. A total of 41 subjects were prospectively recruited in this study, including 11 healthy volunteers and 30 suspected
DVT patients. The healthy volunteers (6 males and 5 females aged 22-54 years old) had no history of DVT and were recruited to evaluate black-blood efficiency and the diagnostic performance of DANTE-FLASH. The suspected DVT patients (19 males and 11 females aged 23-95 years old) were diagnosed from their clinical symptoms, including leg pain, swelling, and positive results on the D-dimer test. No clinical scoring was used, and no other co-morbidities were diagnosed in patients before MR imaging. The patient characteristics are summarized in Table 1 . The patients were classified into 3 groups according to the time from symptom onset to MR scan: 8 patients were in the acute phase ( $\leq 14$ days), 18 were in the subacute phase (15-28 days), and 4 were in the subacute-to-chronic phase (29-180 days) $(15,25)$. The exclusion criteria were known contraindications to MRI and US: (I) standard contraindications to MRI, including claustrophobia, and patients who underwent a surgical procedure within 24 hours before the MRI scan; (II) casts, wounds, or other conditions that caused a US examination unavailable, or obesity or swelling that made the US non-diagnostic.

\section{US examinations}

The suspected DVT patients who had clinical symptoms and positive results on the D-dimer test underwent US examination. The US examination was performed by 


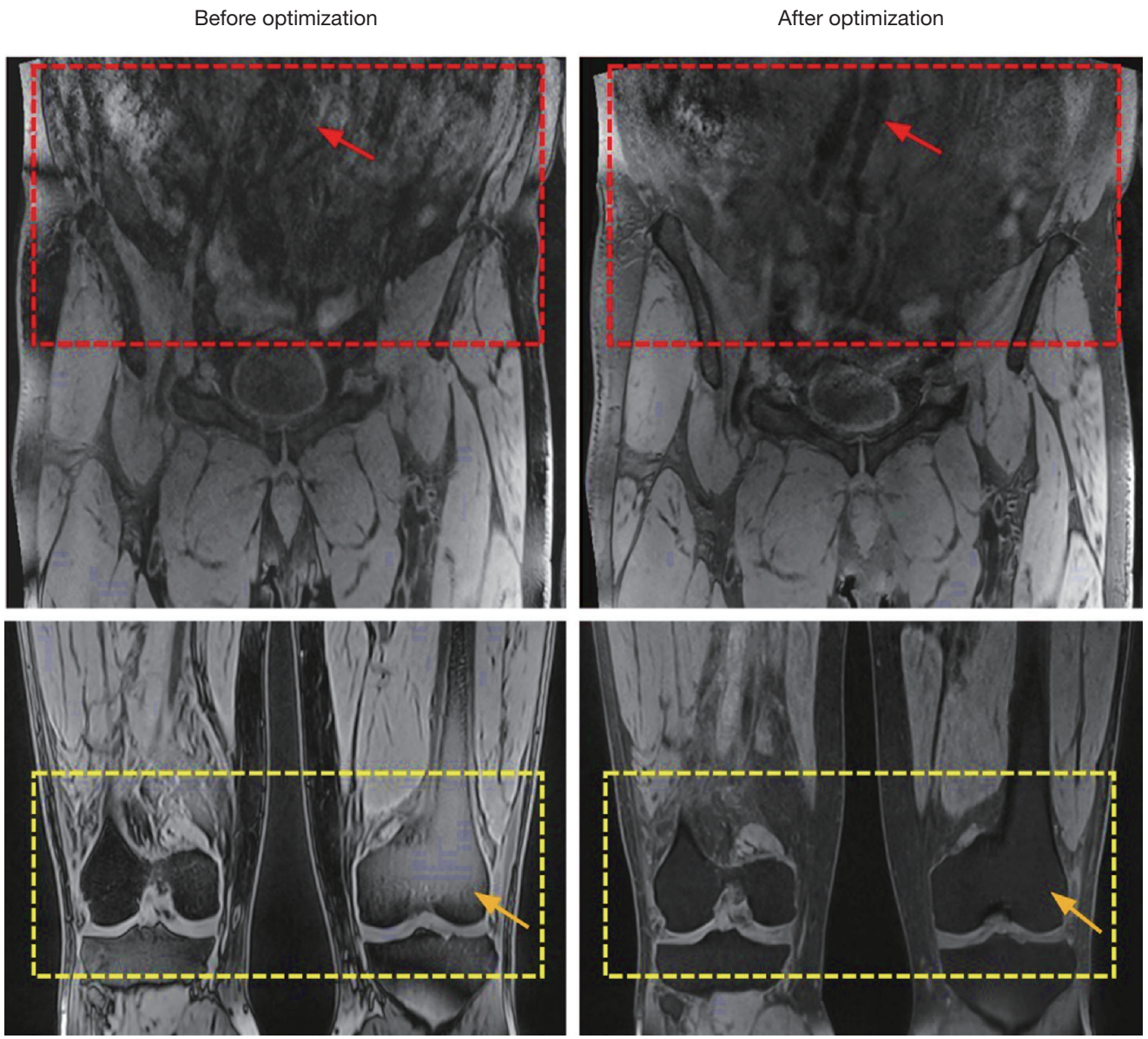

Figure 2 The representative images were obtained by the original and the optimized DANTE-FLASH technique. After technical optimization, DANTE-FLASH realized less abdominal motion artifacts for iliac vessel delineation (red arrows) and achieved more uniform fat saturation (yellow arrows).

a technologist with 15 years of experience who used a standard lower extremity in-depth venous approach (compression and color Doppler US) on a GE system (logic E9) equipped with a 7 - to $10-\mathrm{MHz}$ US probe. Compression and color Doppler US were performed from the common femoral vein to the distal calf, respectively. Only color Doppler US was performed for pelvic veins due to the technical difficulty in detecting pelvic veins using compression US (26). Similar to a previous study (17), the lower extremity deep veins were divided into 3 stacks: the most proximal "iliac stack" included the common iliac veins, the internal iliac veins, and the external iliac veins; the "femoral stack" included the common femoral veins, the femoral veins, and the deep femoral veins; and an additional third "popliteal-crural stack" included the popliteal vein, the tibiofibular trunk veins, the anterior and posterior tibial veins, the fibular vein, and the great and small saphenous veins. The time required to complete the US examination varied between 10 and 30 minutes.

\section{MR imaging}

After the suspected DVT patients were scanned by US, they were then scanned on a $3 \mathrm{~T}$ MR scanner (MAGNETOM Skyra, Siemens Healthcare, Erlangen, Germany) with 2 standard 18-channel body coils and an integrated posterior spine coil. Healthy volunteers also underwent MR imaging to evaluate the diagnostic performance of DANTE-FLASH. Subjects for MR imaging were placed in the feet-first supine position to scan the entire lower limb and underwent 3 -station craniocaudal spatial coverage scanning. The first station was from the common iliac to the common femoral 
Table 1 Patient characteristics

\begin{tabular}{|c|c|}
\hline Characteristic & Values \\
\hline Age, mean \pm SD [range], years & $53.1 \pm 18.3[23-95]$ \\
\hline Male sex, $\mathrm{n}(\%)$ & $19(63.3)$ \\
\hline \multicolumn{2}{|l|}{ Symptoms, n (\%) } \\
\hline Leg pain & $13(43.3)$ \\
\hline Swelling & $18(60.0)$ \\
\hline Relapsing DVT & $1(5.0)$ \\
\hline \multicolumn{2}{|l|}{ DVT stages, n (\%) } \\
\hline Acute stage & $8(26.7)$ \\
\hline Subacute stage & $18(60.0)$ \\
\hline Subacute-to-chronic stage & $4(13.3)$ \\
\hline \multicolumn{2}{|l|}{ MRI } \\
\hline Analyzed vessel segments, $n$ & 780 \\
\hline Iliac segments, n (\%) & $180(23.1)$ \\
\hline Femoral segments, n (\%) & $180(23.1)$ \\
\hline Popliteal-crural segments, n (\%) & $420(53.8)$ \\
\hline $\begin{array}{l}\text { Thrombotic segments with consensus } \\
\text { reading, } \mathrm{n}\end{array}$ & 137 \\
\hline Iliac segments, n (\%) & $31(22.6)$ \\
\hline Femoral segments, n (\%) & $41(29.9)$ \\
\hline Popliteal-crural segments, n (\%) & $65(47.4)$ \\
\hline
\end{tabular}

DVT, deep vein thrombosis; MRI, magnetic resonance imaging.

vein, the second station was from the common femoral to the proximal popliteal vein, and the third station was from the popliteal vein to the distal calf vein. The scan parameters used to perform the DANTE module were as follows: flip angle $=15^{\circ}$, pulse train length $=150$, time delay $=20$ us, and the gradient amplitude $=18 \mathrm{mT} / \mathrm{m}$. The parameters used for the FLASH readout were as follows: echo spacing/ $\mathrm{TE}=5.4 / 2.08 \mathrm{~ms}$, flip angle $=12^{\circ}$, isotropic resolution $=1.2 \times 1.2 \times 1.2 \mathrm{~mm}^{3}$ and reconstruction to $0.6 \times 0.6 \times 0.6 \mathrm{~mm}^{3}$, field of view $(\mathrm{FOV})=380 \times 380 \mathrm{~mm}^{2}$, bandwidth $=$ $425 \mathrm{~Hz} /$ pixel, parallel imaging factor $=2$, and partial Fourier factor $=7 / 8$, and TR $=800 \mathrm{~ms}$. WE was used for fat suppression. Each three-station DANTE-FLASH scan took 10-12 minutes to complete.

Following the DANTE-FLASH scan, MRDTI was also performed as a standard reference. The areas scanned using MRDTI were the same as those scanned using DANTEFLASH to ensure that they had the same scan coverage for comparison. The scan parameters of MRDTI were the same as those used for the FLASH readout in DANTEFLASH except for the inversion recovery time (200 ms) and flip angle $\left(18^{\circ}\right)$. The inversion time and flip angle were optimized to make the blood signal as low as possible and the muscle signal as high as possible consistent with a previous study (27), increasing the possibility that the iso-intense signal thrombus will be identified on MRDTI images. The MRDTI scanning times were the same as those of DANTE-FLASH.

\section{Image analysis}

\section{US examinations}

A radiologist who conducted the US examination interpreted the suspected DVT patients' US images first, then a radiologist with 10 years of US experience conducted verification of the results. DVT was defined as a lack of compressibility and the absence of flow in a venous segment.

\section{MR imaging}

All MR images obtained from the healthy volunteers and patients were loaded onto a workstation (Leonardo; Siemens Healthcare, Germany) for review and analysis.

To quantitatively analyze the image quality and adequate SI of the thrombus on DANTE-FLASH and MRDTI, image signal-to-noise ratio (SNR) and apparent contrastto-noise ratios (CNR) between the muscle and the venous lumen $\left[\left(\mathrm{SI}_{\text {muscle }}-\mathrm{SI}_{\text {lumen }}\right) / \sigma_{\mathrm{n}}\right]$ and between the thrombus and the venous lumen $\left[\left(\mathrm{SI}_{\text {thrombus }}-\mathrm{SI}_{\text {lumen }}\right) / \sigma_{\mathrm{n}}\right]$ were measured. The noise $\sigma_{\mathrm{n}}$ was defined as the standard deviation of the SI as determined in 4 artifact-free background regions to minimize bias due to inhomogeneous signals. SI was measured as the mean SI within a manually drawn region of interest (ROI).

To evaluate the feasibility of using DANTE-FLASH for the diagnosis of DVT, all MR images obtained from healthy volunteers and the suspected DVT patients were randomized for diagnostic review. Two radiologists with 9 and 12 years of cardiovascular MR experience who were blinded to the subjects' information and imaging protocols independently assessed the randomized images. They made a diagnosis of DVT (presence or absence) for each venous segment on both DANTE-FLASH and MRDTI images. All images obtained by DANTE-FLASH were reviewed at the same time. One month later, a consensus reading was performed only on the MRDTI images to generate 
a reference for evaluating the diagnostic performance of DANTE-FLASH. The thrombus was identified as a hyper/ iso-intense signal within the venous lumen on DANTEFLASH images or MRDTI images. Subjective scores for image quality and diagnostic confidence for the 2 techniques across all subjects were independently rated by the same readers on a 4-point scale consistent with previous studies $(15,17)$. Image quality was rated as follows: $4=$ excellent, no relevant artifacts; $3=$ good, minimal inhomogeneity, only minor flow artifacts; $2=$ adequate, delineated lumen, major flow artifacts; and $1=$ insufficient for diagnosis. The diagnostic confidence was also evaluated as follows: $4=$ excellent, exact diagnosis possible; $3=$ good, definite diagnosis possible; 2 = fair, evaluation of major findings possible; and $1=$ poor, definite diagnosis impossible. The diagnostic confidence score was used to rate the confidence of a reader in diagnosing the thrombus, whether it was present or not.

The clot burden was analyzed based on DANTEFLASH and MRDTI images to evaluate the accuracy of DANTE-FLASH for assessing a patient's clot burden. The assessment criteria used to evaluate the clot burden were the same as those used in previous studies $(28,29)$. Specifically, the clot burden of each segment was scored by the radiologists in consensus: 0 , patent vein segment; 1 , nonocclusive thrombus; 2 , subsegmental, occlusive thrombus; and 3, occlusive thrombus of the entire length of a segment. After each segment was assigned a score, the left and right segment scores were then added to obtain a clot burden for a region. The clot burden of a limb was calculated as the summation of all 3 segments in the same limb. The patient clot burden was the summation of all segments.

\section{Statistical analysis}

All statistical analyses were performed using SPSS software (version 17.0, International Business Machines, Armonk, USA). Data are presented as the mean \pm standard deviation (SD). A paired-samples t-test was used to compare image quality and diagnostic confidence scores between DANTEFLASH and MRDTI. The $t$-test was also used for the comparison of SNR and CNR between DANTE-FLASH and MRDTI. Using US, MRDTI, and the consensus reading of MRDTI and US as standard references, the diagnostic SE, SP, PPV, NPV, and ACC values of DANTEFLASH and MRDTI for the diagnosis of DVT patients were calculated. Diagnostic agreements between DANTE-
FLASH and the US and between MRDTI and US, as well as those between DANTE-FLASH and MRDTI, were determined by calculating Cohen's kappa coefficients. The inter-reader diagnostic agreement was also calculated using Cohen's kappa test. Agreement was rated as fair (kappa value $\kappa=0.21-0.40)$, moderate $(\kappa=0.41-0.60)$, substantial $(\kappa=0.61-$ $0.80)$, or excellent $(\kappa>0.80)$ (30). Clot burden was compared between DANTE-FLASH and MRDTI at the region, limb, and patient levels. The Wilcoxon signed-rank test was used to identify differences in clot burden scores (29). A $P$ value less than 0.05 was considered statistically significant.

\section{Results}

For all included subjects, US, DANTE-FLASH, and MRDTI examinations were successfully conducted. In total, 286 vessel segments obtained in 11 healthy volunteers were assessable concurrently by DANTE-FLASH and MRDTI, and 780 vessel segments obtained in 30 suspected DVT patients were assessable concurrently by US, DANTEFLASH, and MRDTI. Two of the suspected DVT patients did not have thrombosis according to the diagnostic results of US, DANTE-FLASH, or MRDTI.

According to the quantitative analysis results, DANTEFLASH achieved higher SNRs for muscle and the thrombus, and higher CNR for muscle vs. venous lumen and thrombus vs. lumen compared to MRDTI (Table 2). DANTE-FLASH achieved better venous lumen delineation compared with MRDTI due to higher contrast between the muscle and the venous lumen (Figure 3). Image quality was rated as excellent by both readers in $43.1 \%(459 / 1,066)$ and $30.4 \%(324 / 1,066)$ of the segments obtained using DANTE-FLASH and MRDTI, respectively. As a result, the overall image quality scores of DANTE-FLASH were significantly higher than that of MRDTI $(3.66 \pm 0.44$ vs. $3.52 \pm 0.52, \mathrm{P}<0.001)$, and the diagnostic confidence of DANTE-FLASH were also higher than that of MRDTI (3.84 \pm 0.36 vs. $3.76 \pm 0.41, \mathrm{P}<0.001)$.

Using the consensus reading of US as the standard reference, higher SE (average values for the 2 readers: $76.5 \%$ vs. $75.0 \%$ ), SP (average values: $90.7 \%$ vs. $90.3 \%$ ), PPV (average values: $57.1 \%$ vs. $56.2 \%$ ), NPV (average values: $96.0 \%$ vs. $95.7 \%$ ), and ACC (average values: $88.7 \%$ vs. $88.2 \%$ ) values were obtained for DANTE-FLASH compared to MRDTI. Both DANTE-FLASH and MRDTI had moderate agreement with US (Table 3). Nevertheless, both DANTE-FLASH and MRDTI achieved excellent 
Table 2 Comparisons of SNR and CNR between DANTE-FLASH and MRDTI

\begin{tabular}{lrrr}
\hline & DANTE-FLASH & MRDTI & P value \\
\hline SNR & & & \\
Muscle & $84.10 \pm 40.96$ & $26.96 \pm 15.33$ & 0.044 \\
Thrombus & $139.91 \pm 93.62$ & $58.69 \pm 23.10$ & 0.000 \\
Venous lumen & $21.59 \pm 12.62$ & $13.53 \pm 9.10$ & 0.013 \\
CNR & & & \\
$\begin{array}{l}\text { Muscle vs. venous } \\
\text { lumen }\end{array}$ & $62.52 \pm 34.33$ & $13.43 \pm 11.85$ & 0.000 \\
$\begin{array}{l}\text { Thrombus vs. } \\
\text { venous lumen }\end{array}$ & $116.27 \pm 85.77$ & $45.27 \pm 23.57$ & 0.002 \\
\hline
\end{tabular}

SNR, image signal-to-noise ratio; DANTE-FLASH, DANTE blackblood preparation in combination with FLASH readout; MRDTI, MR direct thrombus imaging; CNR, contrast-to-noise ratio.
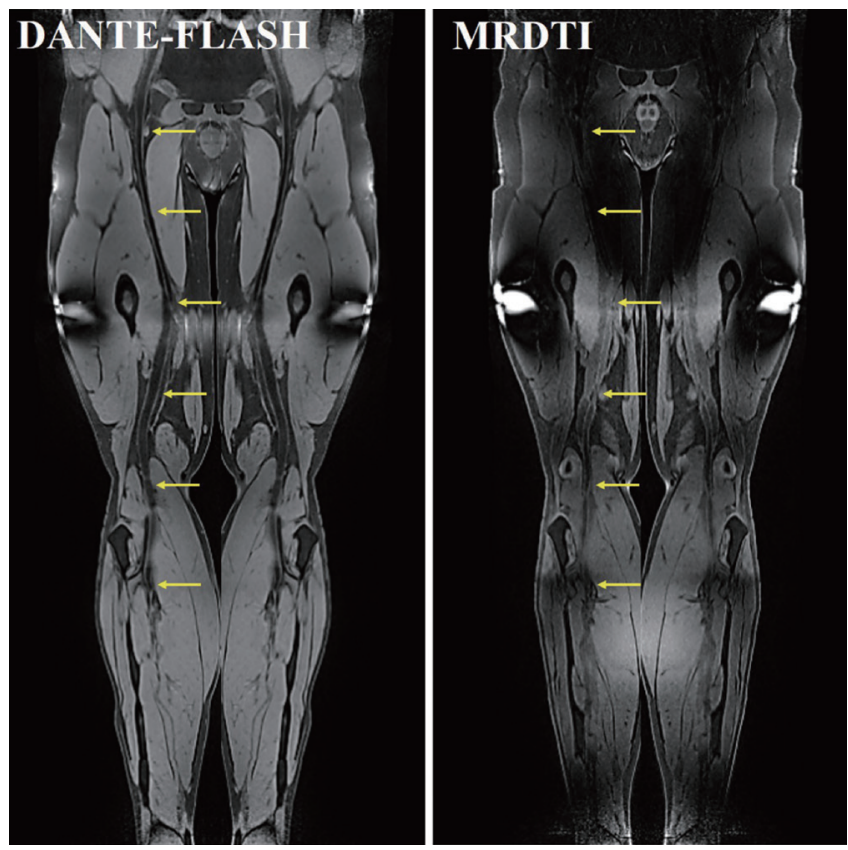

Figure 3 Representative images obtained by DANTE-FLASH and MRDTI in a healthy volunteer. Compared to MRDTI, DANTEFLASH had better blood signal suppression and improved venous lumen delineation (yellow arrows).

interobserver agreement (i.e., $\kappa=0.86, \mathrm{P}<0.001$ and $\kappa=0.84$, $\mathrm{P}<0.001$, respectively) in the diagnosis of DVT.

When all MR images obtained from healthy volunteers and patients were randomized for diagnostic review, a total of 1,066 segments were included for analysis. According to the results of the per-segment based analysis of the MR images, all 11 healthy volunteers and 2 patients without thrombi were correctly excluded from having thrombosis by both DANTE-FLASH and MRDTI. When the consensus reading of MRDTI was used as a standard reference, the diagnostic SE, SP, PPV, NPV, and ACC values of DANTE-FLASH for reader 1 and reader 2 were $94.8 \%$ and $90.3 \%, 98.9 \%$ and $98.0 \%, 92.7 \%$ and $86.4 \%, 99.2 \%$, and $98.6 \%$, and $98.4 \%$ and $97.0 \%$, respectively. DANTEFLASH had excellent diagnostic agreement with MRDTI for both readers $(\kappa=0.93, \mathrm{P}<0.001$ and $\kappa=0.87, \mathrm{P}<0.001$, respectively). The thrombi visualized on DANTE-FLASH images were well-matched to those on MRDTI images (Figure 4). The thrombus can present as hyper-intense, isointense, or the mix of the hyper- and hypo-intense signals on DANTE-FLASH and MRDTI images (Figure 5). It was noted that 2 segments from the external iliac and deep femoral veins were misidentified as having thrombi on MRDTI images because the diagnosis was affected by blood flow artifacts. Also, 2 thrombi located at the external iliac and popliteal veins were missed on MRDTI images by both readers because the thrombi presented as iso-intense signals. Representative images of the blood flow artifacts and iso-intense signal thrombi are shown in Figures 6-8. When the diagnostic results of US were used to correct the misidentified thrombi on MRDTI images, the diagnostic SE, SP, PPV, NPV, and ACC values of DANTE-FLASH for reader 1 and reader 2 were further improved at $97.0 \%$ and $93.2 \%, 99.0 \%$ and $98.2 \%, 93.4 \%$ and $87.9 \%, 99.6 \%$, and $99.0 \%$, and $98.8 \%$ and $97.6 \%$, respectively.

According to the clot burden analysis, the average clot burden scores obtained for DANTE-FLASH and MRDTI were $8.18 \pm 5.81$ and $7.82 \pm 5.66$, respectively. The clot burden was not significantly different between DANTE-FLASH and MRDTI at the region, limb, or patient-level (Table 4).

\section{Discussion}

In this study, DANTE-FLASH was evaluated for its ability to assist in the diagnosis of DVT without the use of a contrast medium. Our experimental results demonstrated that DANTE-FLASH provided time-efficient imaging and excellent thrombus visualization. It had a reliable and accurate diagnostic value for DVT.

The success of DANTE-FLASH in the diagnosis of DVT is due to the DANTE preparation used for blackblood suppression. DANTE can provide excellent blood 
Table 3 The number of thrombosis patients diagnosed on DANTE-FLASH, MRDTI, and the US among the suspected DVT patients

\begin{tabular}{|c|c|c|c|c|}
\hline Vessel segment & Category & $\begin{array}{l}\text { DANTE-FLASH: } \\
\text { reader 1/reader } 2\end{array}$ & $\begin{array}{c}\text { MRDTI: } \\
\text { reader 1/reader } 2\end{array}$ & $\begin{array}{l}\text { US: consensus } \\
\text { reading }\end{array}$ \\
\hline Iliac vessel segment & Thrombosis & $31 / 31$ & $27 / 30$ & 6 \\
\hline Femoral vessel segment & Thrombosis & $41 / 44$ & $42 / 47$ & 30 \\
\hline Popliteal-crural vessel segment & No thrombosis & $355 / 355$ & $358 / 349$ & 316 \\
\hline 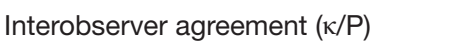 & & $0.86 /<0.001$ & $0.84 /<0.001$ & \\
\hline Diagnostic agreement with US $(\kappa / P)$ & & $0.73 /<0.001 / 0.65 /<0.001$ & $0.71 /<0.001 / 0.63 /<0.001$ & NA \\
\hline
\end{tabular}

DANTE-FLASH, DANTE black-blood preparation in combination with FLASH readout; DVT, deep vein thrombosis; MRDTI, MR direct thrombus imaging; the US, ultrasound.
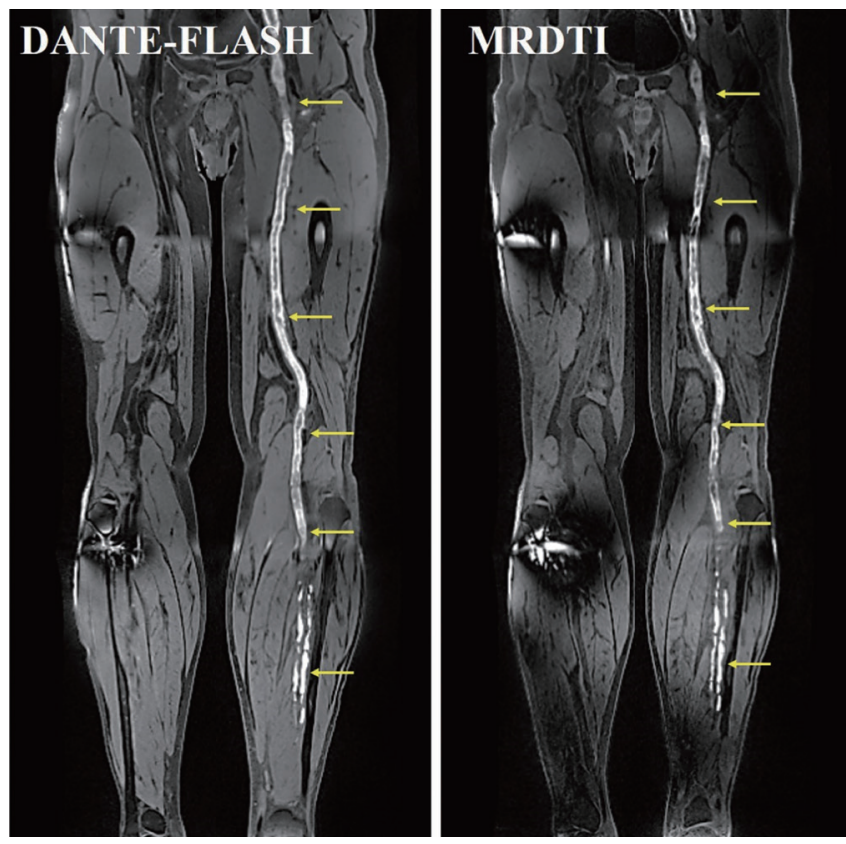

Figure 4 Example images obtained in a 60-year-old deep vein thrombosis (DVT) patient. The thrombus detected by DANTEFLASH was a good match with the MRDTI-detected thrombus (yellow arrows).

flow signal suppression with only approximately $15 \%$ signal attenuation in static tissues, and it has therefore been demonstrated to be a suitable black-blood preparation module for thrombus imaging $(15,16)$. Because DANTE was combined with a centric-ordered FLASH readout in the DANTE-FLASH technique, the venous blood had a rather hypo-intense appearance while thrombi had relative hyperintense signals within the venous lumen. Thus, a thrombus, even an iso-intense thrombus, can be directly visualized and readily identified within the dark venous lumen. The use of a 3D FLASH readout allowed for both time-efficient and low SAR imaging (18,31). In this study, the three-station scan of DANTE-FLASH took only 10 12 minutes to cover the whole bilateral lower limb region at a $3 \mathrm{D}$ isotropic spatial resolution of $1.2 \times 1.2 \times 1.2 \mathrm{~mm}^{3}$, and it is, therefore, feasible to apply DANTE-FLASH in clinical patient scans.

It should be noted that different intensity signals of the thrombi were observed on DANTE-FLASH and MRDTI images. This is because the formation of blood clots can produce methemoglobin, which contains $\mathrm{Fe}^{3+}$. The paramagnetic properties of $\mathrm{Fe}^{3+}$ in methemoglobin can shorten the T1 relaxation time of the thrombus, which makes the thrombus show a relatively high-intensity signal. A previous study demonstrated that the content of $\mathrm{Fe}^{3+}$ contained in the thrombus varies along with the evolution of DVT (12). The variations in $\mathrm{Fe}^{3+}$ content in the thrombus manifest as hyper-intense, hypo-intense, and the mix of hyper- and hypo-intense signals on T1-weighted images (Figure 5).

Using the US as the reference, reader 1 misinterpreted 36 segments, and reader 2 misinterpreted 35 segments as false negatives on DANTE-FLASH images. Of these, 21 segments were in the iliac vein, 9 segments for reader 1 and 12 segments for reader 2 in the femoral veins, and 6 segments for reader 1 and 12 segments for reader 2 were in the popliteal-crural veins, resulting in an overall SE of 

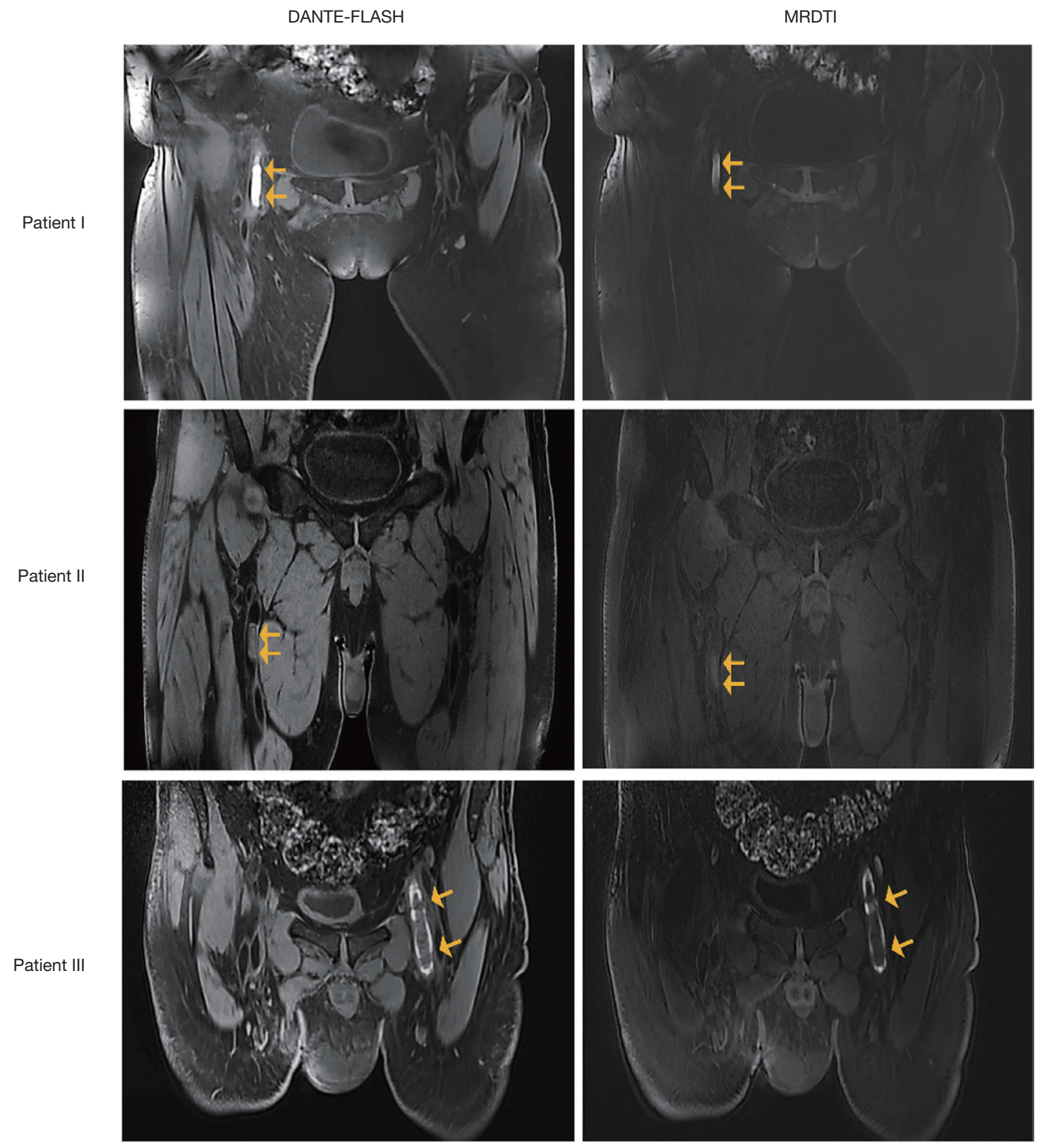

Figure 5 Thrombi (yellow arrows) with different signal intensities from 3 patients. Hyper-intense (patient I), iso-intense (patient II), and the mix of hyper- and hypo-intense signals (patient III) were observed on DANTE-FLASH and MRDTI images.

$76.5 \%$ for DANTE-FLASH in detecting DVT. Because the US is the first-line imaging modality for DVT detection, the diagnostic performance of DANTE-FLASH was first evaluated using the US as the standard reference. However, previous studies demonstrated that the accuracy of the US is considerably lower for the diagnosis of distal DVT (32), in which there is a high chance of false-negative findings due to patient differences and variability in the distal veins (33).
The SE of the US for the detection of DVT, therefore, varies according to the location of the thrombus, ranging from $94.2 \%$ for proximal to $63.5 \%$ for distal veins (34). Our study results also demonstrate that DANTE-FLASH ( $84.7 \%$ vs. $71.8 \%)$ and MRDTI $(82.0 \%$ vs. $71.0 \%)$ provided better performance for proximal veins than for distal veins when the US was used as the standard reference.

When the consensus MRDTI was used as the reference, 

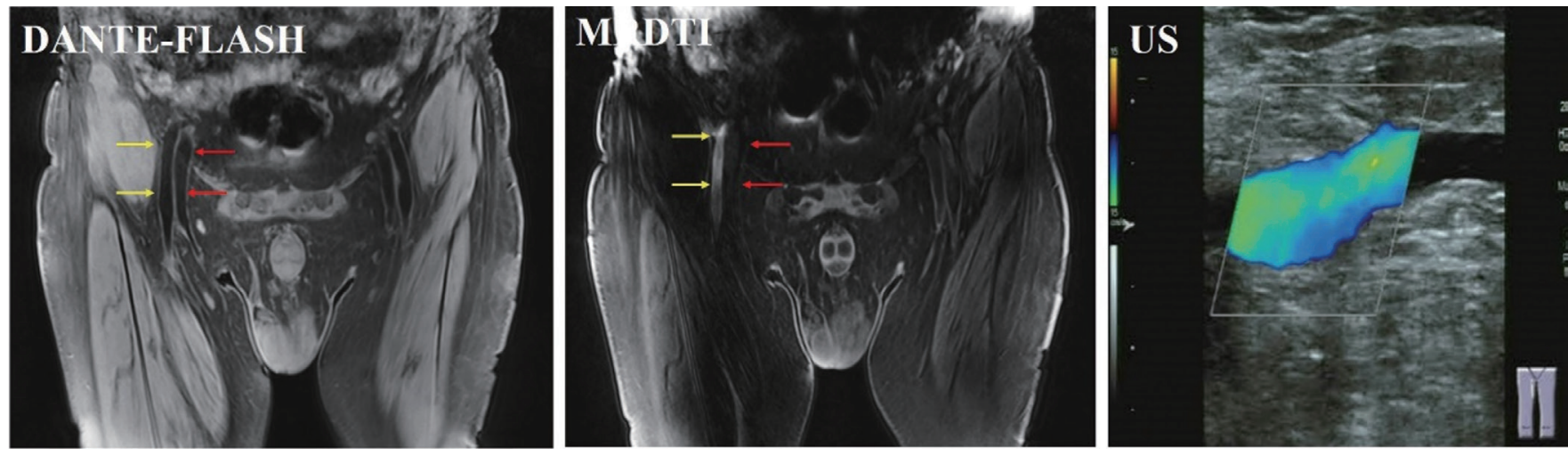

Figure 6 Example images obtained in a deep vein thrombosis (DVT) patient. Arterial (yellow arrows) and venous (red arrows) blood flow artifacts were observed, and the venous segment was misidentified as a thrombus on MRDTI. DANTE-FLASH effectively eliminated the blood flow artifacts, and the venous blood flow was confirmed by ultrasound (US).
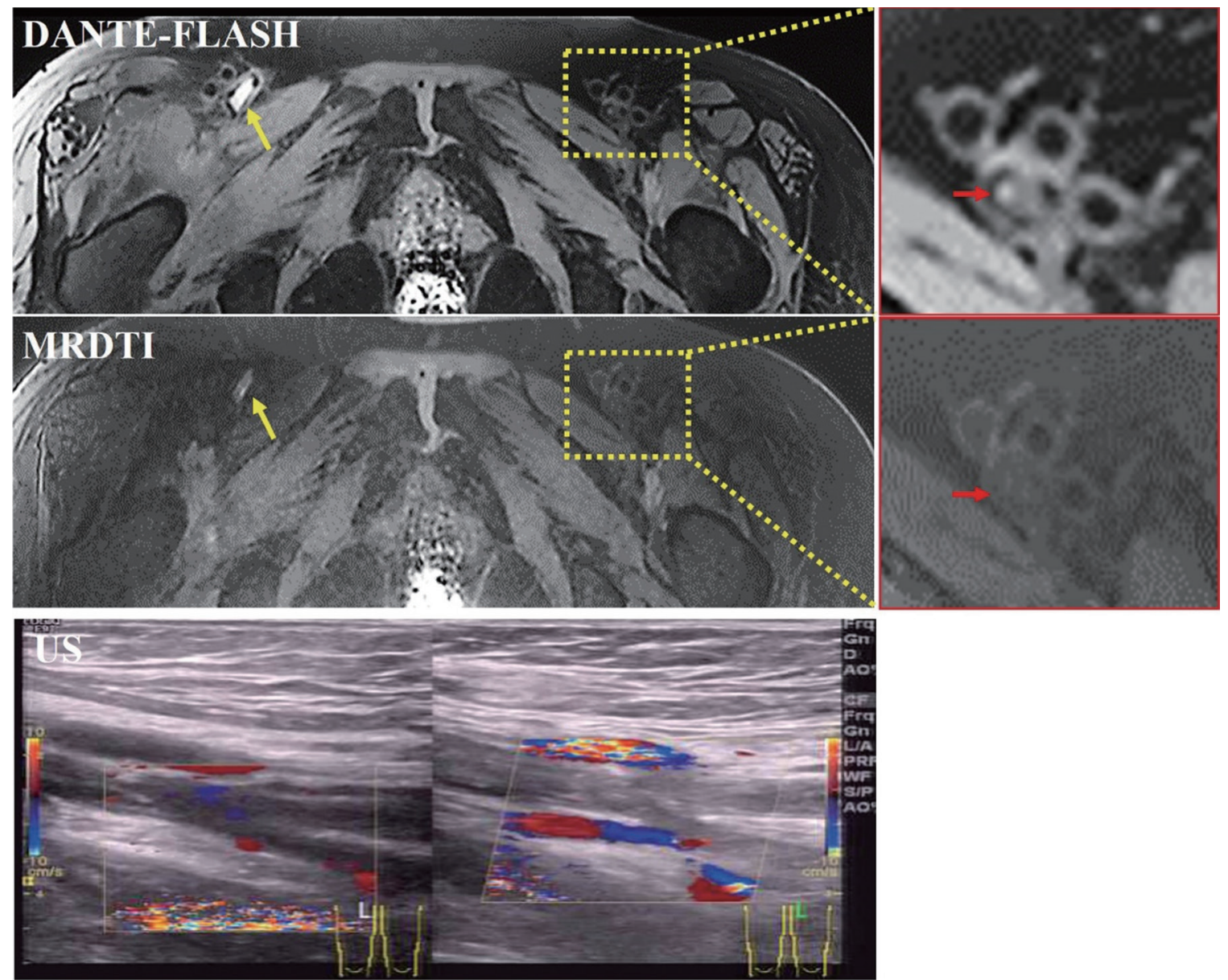

Figure 7 Example images obtained in a deep vein thrombosis (DVT) patient. Both DANTE-FLASH and MRDTI easily identified a hyperintense thrombus (yellow arrows). However, the iso-intense thrombus (red arrows) was missed by both readers on MRDTI images but was identified on DANTE-FLASH images. This was because the SNR of muscle was much higher, and the CNR was higher between the thrombus and venous lumen on DANTE-FLASH compared with MRDTI. 

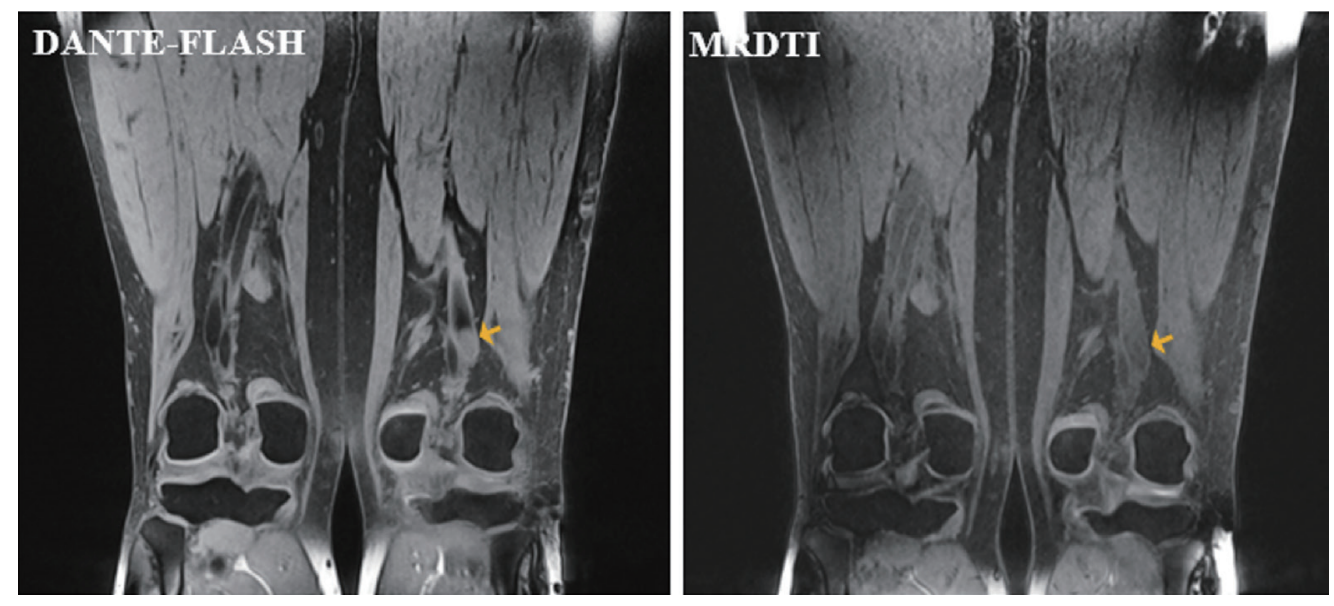

Figure 8 Representative images of iso-intense signal thrombi that were present on DANTE-FLASH images but were missed on MRDTI images by both readers (yellow arrows).

Table 4 Comparison of clot burden between DANTE-FLASH and MRDTI

\begin{tabular}{lccc}
\hline Regions & DANTE-FLASH & MRDTI & P value \\
\hline lliac vessel segment & $0.34 \pm 0.89$ & $0.33 \pm 0.87$ & 0.152 \\
$\begin{array}{l}\text { Femoral vessel } \\
\text { segment }\end{array}$ & $0.47 \pm 0.91$ & $0.46 \pm 0.90$ & 0.326 \\
$\begin{array}{l}\text { Popliteal-crural vessel } \\
\text { segment }\end{array}$ & $0.23 \pm 0.62$ & $0.22 \pm 0.59$ & 0.181 \\
Limb & $4.09 \pm 5.50$ & $3.91 \pm 5.29$ & 0.392 \\
Patients & $8.18 \pm 5.81$ & $7.82 \pm 5.66$ & 0.315 \\
\hline
\end{tabular}

DANTE-FLASH, DANTE black-blood preparation in combination with FLASH readout; MRDTI, MR direct thrombus imaging.

readers 1 and 2 detected 7 and 13 thrombi as falsenegative vessel segments on DANTE-FLASH images. These included 2 segments for reader 1 and 1 for reader 2 in the iliac veins, 3 segments for reader 2 in the femoral vein, and 5 segments for reader 1 and 9 segments for reader 2 in the popliteal-crural veins. There are 3 main reasons for the observed differences in the interpretations of DANTE-FLASH and MRDTI images. First, the low contrast between the muscle and venous lumen observed on MRDTI images and the complex vessel anatomy of the lower extremities make it challenging to identify the correct venous vessels on MRDTI images. Both readers held different views regarding thrombi located at the internal or external iliac segments and the femoral veins or deep femoral veins.
Similarly, a thrombus located in the intermuscular vein of the calf on the MRDTI images was identified as being located in the fibular vein in the DANTE-FLASH images. Second, due to the lower contrast between the isointense thrombus and the venous lumen when observed on MRDT1 images, the iso-intense thrombus can be easily missed by readers (Figures 7 and 8). In this study, 4 isointense thrombi were identified by DANTE-FLASH, and 3 of them were missed on MRDTI. Third, MRDTI contains more severe blood flow artifacts than DANTE-FLASH, and these blood flow artifacts could be misidentified as a thrombus by readers (Figure 6). When the consensus results of US and MRDTI were the standard references, the diagnostic performance of DANTE-FLASH was improved. Nevertheless, the diagnostic performance of DANTEFLASH had an excellent agreement with those found on MRDTI, and thus, the diagnostic SE, SP, PPV, NPV, and ACC values were similar between DANTE-FLASH and MRDTI using the US as a standard reference.

The clinical symptoms of DVT and the D-dimer test are not specific to DVT, thus suspected DVT patients should undergo an imaging scan as part of the final diagnostic decision (35-38). In this study, the suspected DVT patients who had clinical symptoms and positive results on the D-dimer test were subjected to US and MR examinations. Two of the suspected DVT patients were excluded from having thrombosis by all 3 imaging modalities used in our study. After checking the patients' information, we found that these 2 patients were hospitalized and had been treated by additional catheter-directed thrombolysis. 
This demonstrated that the thrombi of the 2 patients were successfully removed after treatment, highlighting DANTE-FLASH's accuracy for DVT diagnosis.

It should be noted that both DANTE-FLASH and MRDTI resulted in susceptibility artifacts at the corners of each scan station. This is because, in this study, a large FOV $\left(380 \times 380 \mathrm{~mm}^{2}\right)$ was used to cover a large area, and WE was used for fat saturation. The large FOV made B0 shimming difficult and resulted in B0 field inhomogeneity, especially at the corners of the FOV. Although WE is a suitable technique for achieving homogeneous fat suppression in a large FOV, it is still affected by an inhomogeneous B0 field which results in artifacts arising at the corners of each scan station (22). Fortunately, the artifacts had little effect on the identification of the thrombus; hence we preferred to use a large FOV to achieve extensive coverage.

It should also be noted that DANTE-FLASH has been introduced for vessel wall imaging in previous work (18). Although DANTE-FLASH used for vessel wall imaging also utilizes the DANTE preparation and FLASH readout, the technique needs to be optimized for thrombus imaging to provide more uniform fat suppression and fewer abdomen artifacts (Figure 2). In addition, as blood flow in the veins is much slower than in the arteries, whether the DANTE preparation can sufficiently suppress venous blood flow and achieve reliable thrombus imaging remains unclear. Thus, further investigation is warranted to optimize the DANTEFLASH technique and investigate its feasibility for the diagnosis of DVT.

There are several limitations to this study. First, digital subtraction angiography (DSA), which is the gold standard technique, was not conducted for comparison. This is because DSA is an interventional and ionizing radiation technique. Furthermore, it is seldom used for diagnosing DVT nowadays. Thus, the US, which is the first-line diagnostic technique for DVT diagnosis, and MRDTI, which has been shown to diagnose DVT accurately, were used as standard references for comparison. Second, DANTE-FLASH was not compared to current BTI techniques. We did not compare it is because if BTI were also conducted in this study, it would take more than half an hour to complete all $3 \mathrm{MR}$ techniques (e.g., MRDTI, DANTE-FLASH, and BTI). It was not feasible to fit such an excessively long study into the current schedule. Third, the number of recruited suspected DVT patients was only 30. A larger patient population is needed to evaluate this technique in future studies. Nevertheless, it should be noted that the data analysis was based on a segment level, not a patient level. The total number of vessel segments was 1,066 for data analysis and statistics. This number is sufficient, and potential sampling bias has little effect on the data analysis. Finally, follow-up of patients after treatment is necessary for further evaluating the clinical value of DANTE-FLASH. However, this study was focused on evaluating whether DANTE-FLASH can be used for the diagnosis of DVT. Thus, we did not perform follow-up evaluations in this study.

In conclusion, DANTE-FLASH is a time-efficient and contrast-free imaging technique that accurately detects thrombi and provides better image quality than MRDTI. It may, therefore, serve as a safe and convenient alternative for the diagnosis of DVT when the US cannot be performed.

\section{Acknowledgments}

Funding: This project was supported in part by the National Natural Science Foundation of China (81971607, 81729003), the Natural Science Foundation of Guangdong (2017A050501026), and the Natural Science Foundation of Shenzhen (JCYJ20160331185933583, GJHZ20150316143320494, KQCX2015033117354154).

\section{Footnote}

Conflicts of Interest: All authors have completed the ICMJE uniform disclosure form (available at http://dx.doi. org/10.21037/qims-19-761). Dr. XB reports other from Siemens, outside the submitted work. Dr. GX reports grants from the National Natural Science Foundation of China, grants from the Natural Science Foundation of Guangdong, and grants from the Natural Science Foundation of Shenzhen during the conduct of the study. The other authors have no conflicts of interest to declare.

Ethical Statement: The local institutional review board approved this study, and written informed consent was obtained from all participants.

Open Access Statement: This is an Open Access article distributed in accordance with the Creative Commons Attribution-NonCommercial-NoDerivs 4.0 International License (CC BY-NC-ND 4.0), which permits the noncommercial replication and distribution of the article with the strict proviso that no changes or edits are made and the original work is properly cited (including links to both the formal publication through the relevant DOI and the license). 
See: https://creativecommons.org/licenses/by-nc-nd/4.0/.

\section{References}

1. Spencer FA, Emery C, Lessard D, Anderson F, Emani S, Aragam J, Becker RC, Goldberg RJ. The worcester venous thromboembolism study. J Gen Intern Med 2006;21:722-7.

2. Goldhaber SZ. Pulmonary Embolism. New Engl J Med 1998;339:93-104.

3. Zheng MZ, Yang Q, Lu X, Hu S, Chai C, Shen W, Chang B, Wang Z, Xia S. Middle cerebral artery thrombus susceptibility-weighted imaging mapping predicts prognosis. Quant Imaging Med Surg 2019;9:1556-65.

4. Karande GY, Hedgire SS, Sanchez Y, Baliyan V, Mishra V, Ganguli S, Prabhakar AM. Advanced imaging in acute and chronic deep vein thrombosis. Cardiovasc Diagn Ther 2016;6:493-507.

5. Zitek JA, Baydoun J, Baird J. Tools for the Clinician: The Essentials of Bedside (ED or ICU) Ultrasound for Deep Vein Thrombosis. Curr Emerg Hosp Med Rep 2013;1:65-70.

6. Redman HC. Deep venous thrombosis: is contrast venography still the gold standard? Radiology 1988;168:277-8.

7. Zierler BK. Ultrasonography and Diagnosis of Venous Thromboembolism. Circulation 2004;109:19-14.

8. Stevens SM, Fazili M, Woller SC. Choosing ultrasound technique for suspected deep vein thrombosis: which is best? Quant Imaging Med Surg 2020;10:1418-21.

9. Divittorio R, Bluth EI, Sullivan MA. Deep vein thrombosis: Diagnosis of a comon clinical problem. Ochsner J 2002;4:14-7.

10. Sampson FC, Goodacre SW, Thomas SM, van Beek EJ. The accuracy of MRI in diagnosis of suspected deep vein thrombosis: systematic review and meta-analysis. Eur Radiol 2007;17:175-81.

11. Sadowski EA, Bennett LK, Chan MR, Wentland AL, Garrett AL, Garrett RW, Djamali A. Nephrogenic systemic fibrosis: risk factors and incidence estimation. Radiology 2007;243:148-57.

12. Moody AR. Magnetic resonance direct thrombus imaging. J Thromb Haemost 2003;1:1403-9.

13. Ono A, Murase KT, Shibutani O, Takata S, Kobashi Y, Miyazaki M. Deep vein thrombosis using noncontrastenhanced MR venography with electrocardiographically gated three-dimensional half-Fourier FSE: preliminary experience. Magn Reson Med 2009;61:907-17.
14. Young IR, Szeverenyi NM, Du J, Bydder GM. Pulse sequences as tissue property filters (TP-filters): a way of understanding the signal, contrast and weighting of magnetic resonance images. Quant Imaging Med Surg 2020;10:1080-120.

15. Xie G, Chen H, He X, Liang J, Deng W, He Z, Ye Y, Yang Q, Bi X, Liu X, Li D, Fan Z. Black-blood thrombus imaging (BTI): a contrast-free cardiovascular magnetic resonance approach for the diagnosis of non-acute deep vein thrombosis. J Cardiovasc Magn Reson 2017;19:4.

16. Chen H, He X, Xie G, Liang J, Ye Y, Wei D, He Z, Liu D, Li D, Xin L. Cardiovascular magnetic resonance blackblood thrombus imaging for the diagnosis of acute deep vein thrombosis at 1.5 Tesla. J Cardiovasc Magn Reson 2018;20:42.

17. Treitl KM, Treitl M, Kooijman-Kurfuerst H, Kammer NN, Coppenrath E, Suderland E, Czihal M, Hoffmann U, Reiser MF, Saam T. Three-Dimensional Black-Blood T1Weighted Turbo Spin-Echo Techniques for the Diagnosis of Deep Vein Thrombosis in Comparison With ContrastEnhanced Magnetic Resonance Imaging: A Pilot Study. Invest Radiol 2015;50:401-8.

18. Xie G, Zhang N, Xie Y, Nguyen C, Deng Z, Bi X, Fan Z, Liu X, Li D, Fan Z. DANTE-prepared 3D FLASH: A fast isotropic-resolution MR approach to morphological evaluation of the peripheral arterial wall at 3 Tesla. J Magn Reson Imaging 2016;43:343-51.

19. Li L, Miller KL, Jezzard P. DANTE-prepared pulse trains: A novel approach to motion-sensitized and motionsuppressed quantitative magnetic resonance imaging. Magn Reson Med 2012;68:1423-38.

20. Li L, Chai JT, Biasiolli L, Robson MD, Choudhury RP, Handa AI, Near J, Jezzard P. Black-Blood Multicontrast Imaging of Carotid Arteries with DANTE-prepared 2D and 3D MR Imaging. Radiology 2014;273:560-9.

21. Andrews B, Sommerville K, Austin S, Wilson N, Browse NL. Effect of foot compression on the velocity and volume of blood flow in the deep veins. Brit J Surg 1993;80:198-200.

22. Hauger O, Dumont E, Chateil JF, Moinard M, Diard F. Water Excitation as an Alternative to Fat Saturation in MR Imaging: Preliminary Results in Musculoskeletal Imaging. Radiology 2002;224:657-63.

23. Sala E, Graves M, Jardine V, Joubert I, Lomas D. Abdominal MRI: evaluation of binomial water excitation for fat suppression. In Proc Intl Soc Mag Reson Med 2003;11:2003.

24. Del Grande F, Santini F, Herzka DA, Aro MR, Dean CW, Gold GE, Carrino JA. Fat-Suppression Techniques 
for 3-T MR Imaging of the Musculoskeletal System. Radiographics 2014;34:217-33.

25. Vedantham S, Grassi CJ, Ferral H, Patel NH, Thorpe PE, Antonacci VP, Janne D'Othée BM, Hofmann LV, Cardella JF, Kundu S, Lewis CA, Schwartzberg MS, Min RJ, Sacks D. Reporting Standards for Endovascular Treatment of Lower Extremity Deep Vein Thrombosis. J Vasc Interv Radiol 2006;17:417-34.

26. Mumoli N, Vitale J, Giorgi-Pierfranceschi M, Sabatini S, Tulino R, Cei M, Bucherini E, Bova C, Mastroiacovo D, Camaiti A. General Practitioner-Performed Compression Ultrasonography for Diagnosis of Deep Vein Thrombosis of the Leg: A Multicenter, Prospective Cohort Study. Ann Fam Med 2017; 15:535-9.

27. Priest AN, Joubert I, Hilborne S, Hunter S, Bowden DJ, Graves MJ. MR Direct Thrombus Imaging with optimised signal and improved lipid suppression. Proceedings of 21 st Annual Meeting of ISMRM,Salt Lake City, Utah, USA; 2013:1280.

28. Protack CD, Bakken AM, Patel N, Saad WE, Waldman DL, Davies MG. Long-term outcomes of catheter directed thrombolysis for lower extremity deep venous thrombosis without prophylactic inferior vena cava filter placement. J Vasc Surg 2007;45:992-7.

29. Wu G, Xie R, Zhang X, Morelli J, Yan X, Zhu X, Li $X$. The Diagnostic Value of 3-Dimensional Sampling Perfection With Application Optimized Contrasts Using Different Flip Angle Evolutions (SPACE) MRI in Evaluating Lower Extremity Deep Venous Thrombus. Invest Radiol 2017;52:734-40.

30. Landis JR, Koch GG. The measurement of observer agreement for categorical data. Biometrics 1977;33:159-74.

31. Balu N, Yarnykh VL, Chu B, Wang J, Hatsukami T, Yuan C. Carotid plaque assessment using fast 3D

Cite this article as: Mao $\mathrm{H}$, Guan X, Peng K, Cai Y, Yang J, He X, Chen H, Zhang X, Bi X, Liu X, Li D, Fan Z, Deng Z, Xie G. Time-efficient and contrast-free magnetic resonance imaging approach to the diagnosis of deep vein thrombosis on black-blood gradient-echo sequence: a pilot study. Quant Imaging Med Surg 2021;11(1):276-289. doi: 10.21037/qims-19-761 isotropic resolution black-blood MRI. Magn Reson Med 2011;65:627-37.

32. Cogo A, Lensing AW, Wells P, Prandoni P, Büller HR. Noninvasive objective tests for the diagnosis of clinically suspected deep-vein thrombosis. Haemostasis 1995;25:27-39.

33. Huisman MV, Klok FA. Diagnostic management of acute deep vein thrombosis and pulmonary embolism. J Thromb Haemost 2013;11:412-22.

34. Goodacre S, Sampson F, Thomas S, van Beek E, Sutton A. Systematic review and meta-analysis of the diagnostic accuracy of ultrasonography for deep vein thrombosis. BMC Med Imaging 2005;5:6.

35. Wells PS, Hirsh J, Anderson DR, et al. Accuracy of clinical assessment of deep-vein thrombosis. Lancet 1995;345:1326-30.

36. Ho VB, van Geertruyden PH, Yucel EK, Rybicki FJ, Baum RA, Desjardins B, Flamm SD, Foley WD, Jaff MR, Koss SA, Mammen L, Mansour MA, Mohler ER, Narra VR, Schenker MP. ACR Appropriateness Criteria(®) on suspected lower extremity deep vein thrombosis. J Am Coll Radiol 2011;8:383-7.

37. Tan M, Mol GC, Ree MAVD, Rooden CJV, Westerbeek RE, Sol AID, Roos AD, Huisman MV. Accuracy of Magnetic Resonance Direct Thrombus Imaging (MRDTI) As a Novel Tool in the Diagnosis of Acute Ipsilateral Recurrent Deep Vein Thrombosis. Blood 2012;120:395.

38. Tan M, Mol GC, van Rooden CJ, Klok FA, Westerbeek RE, Iglesias Del Sol A, van de Ree MA, de Roos A, Huisman MV. Magnetic resonance direct thrombus imaging differentiates acute recurrent ipsilateral deep vein thrombosis from residual thrombosis. Blood 2014;124:623-7. 